Trab. Ling. Aplic., Campinas, (43): 51-68, Jan./Jun. 2004

\title{
NEGOCIANDO CERTEZA E INCERTEZA EM ESCRITA ACADÊMICA EM INGLÊS
}

\author{
LEONARDO RECSKI* \\ (Universidade Federal de Santa Catarina)
}

\begin{abstract}
Expressing claims with an adequate degree of certainty and uncertainty is one of the major setbacks for foreign language students writing academic essays in English. In order to differentiate beliefs from facts and evaluate statements in satisfactory and persuasive ways authors have to make use of epistemic comments which exert a crucial role in academic writing. However, regardless of its significance, little is known about how foreign language learners present their claims in their writing. This article compares exponents of doubt and certainty in the essays of 67 Brazilian speaking university students writing in English with those of 52 British and American learners of similar age and educational level in a corpus of about 68,000 words. A meticulous analysis of the essays reveals that the Brazilian writers deviate considerably from their native speaker counterparts in employing a more restricted variety of items, being much more assertive in relation to their propositions and demonstrating greater difficulties in transmitting an exact degree of assurance. This paper stresses a number of issues raised by the study and makes some pedagogical recommendations for developing skills in this central pragmatic area. Keywords: Academic essay writing, EFL, expressing doubt and certainty.
\end{abstract}

\section{INTRODUÇÃO}

A habilidade de expressar apropriadamente certeza ou incerteza em inglês é uma tarefa complexa para aprendizes de English as a Foreign Language (EFL), entretanto, tal habilidade constitui um elemento fundamental para uma escrita acadêmica bem sucedida. Para serem eficientes, escritores precisam fazer proposições que sejam julgadas adequadas por seus leitores e que reflitam interações sociais adequadas. Uma proposição deve não apenas indicar o quanto o escritor acredita na sua veracidade, que pode variar de remota possibilidade a um prognóstico confiante, mas também indicar um grau de confiança e modéstia aceitáveis por seus leitores. Estes aspectos do uso da linguagem são geralmente referidos como epistêmicos: eles possibilitam que escritores expressem seus julgamentos acerca de possibilidades e indicam um grau de confiança naquilo que dizem (Coates, 1987:112). Tais comentários são geralmente vistos como uma das principais ferramentas utilizadas por escritores para usar a linguagem de maneira flexível para adotar

"Agradecimentos: Gostaria de agradecer a Hamilton Wielewicki por seus pertinentes comentários e grande ajuda na edição deste artigo. 
posicionamentos, expressar pontos de vista e sinalizar simpatia e/ou antipatia (Lyons, 1977; Stubbs, 1986).

Enquanto essas formas podem ter um efeito considerável sobre a maneira pela qual leitores interpretam aspectos referenciais e afetivos em textos, elas são, reconhecidamente, difíceis de serem adquiridas por aprendizes de EFL. Isto se deve não apenas ao fato de que o comprometimento do escritor pode ser expresso através de inúmeras formas, mas também porque essas expressões podem trazer consigo uma variedade muito grande de significados. Outro problema enfrentado por esses aprendizes é o fato de que os esforços empreendidos por eles para dominar tais expressões são geralmente comparados a padrões muito além de suas capacidades, padrões tais como artigos acadêmicos, um gênero que geralmente passa por rigorosa revisão antes de ser publicado. Como observa Hamp-Lyons (1991:57), aprendizes são freqüentemente avaliados com base nas normas de uma comunidade acadêmica na qual eles talvez tenham pouca experiência ou à qual eles talvez ainda não pertençam. Certamente, nem todos os escritores nativos são, da mesma forma, competentes na manipulação de aspectos retóricos quando se trata de textos argumentativos. Logo, enquanto professores necessitam de uma boa compreensão de escrita em EFL para que possam nortear seus aprendizes de forma mais eficiente, ainda não dispomos de informações empíricas suficientes sobre como aprendizes de diferentes línguas diferem nas maneiras pelas quais expressam certeza ou incerteza em inglês.

Este artigo busca indicar como essa importante área de competência pragmática é expressa em composições produzidas por aprendizes de EFL através da investigação de itens lexicais usados tanto para diminuir quanto para intensificar o comprometimento de escritores em dois pequenos corpora de textos escritos. $\mathrm{O}$ artigo investiga como aprendizes de EFL brasileiros modificam suas proposições em composições argumentativas e se estas diferem das estratégias empregadas por um grupo de alunos britânicos e americanos com idade e nível escolar semelhantes. O objetivo desse artigo é, portanto, determinar as formas e significados tipicamente selecionados por estes dois grupos para apresentar seus pontos de vista e suas proposições em escrita acadêmica em inglês.

\section{A COMPLEXIDADE DE EXPRESSÕES MODAIS}

A expressão de certeza e incerteza é fundamental para as convenções de escrita acadêmica em inglês, onde se espera que escritores ofereçam uma avaliação da informação apresentada através de comentários sobre sua provisoriedade ou definitividade. Muitos estudos têm demonstrado a importância pragmática da modalidade como um recurso discursivo para a negociação de conhecimento assim como um veículo para que se possa expressar pontos de vista em relação a proposições (Holmes, 1988; Vande-Kopple, 1995; Crismore, 1990; Biber et al., 1998; Salager-Meyer, 1994, 1998, 2000; Channel, 1994; Halliday, 1994). A construção de estruturas argumentativas adequadas é uma tarefa árdua para aprendizes de EFL e, estes, compreensivelmente, têm dificuldades em qualificar o relacionamento entre suas evidências e a forma como apresentam proposições em relação a elas. 
Expressões modais são complexas para aprendizes por serem polipragmáticas, ou seja, elas podem simultaneamente transmitir diferentes significados. É difícil estabelecer uma correspondência exata e unívoca entre formas em particular e funções específicas, porque um único item lexical, como por exemplo could, pode expressar habilidade ou permissão, assim como possibilidade (Coates, 1983; Palmer, 1990). Certos itens podem, portanto, ser compreendidos como expressões de certeza ou incerteza, de acordo com o contexto onde ocorrem. Os significados não residem nos itens em si, mas são atribuídos às orações que os contém. A determinação de como um item é empregado, portanto, requer uma interpretação pragmática de exemplos autênticos da linguagem.

O uso pragmático de dispositivos epistêmicos é deveras complexificado pelo fato de que eles não apenas transmitem a confiança do escritor na veracidade da informação que está sendo referenciada, mas também ajudam a estabelecer um relacionamento com o leitor. Esta dimensão afetiva envolve uma necessidade de cooperação e consideração. O que se espera de escritores é que reduzam a definitividade ${ }^{1}$ de suas afirmações para que, com isso, possam assegurar a ratificação das mesmas (Holmes, 1984; Hyland, 1996c). Declarações de certeza absoluta, ou afirmações categóricas não deixam espaço para feedback e relegam ao leitor um papel passivo. O uso de dispositivos lingüísticos que reduzam a força de uma afirmação, portanto, faz com que o escritor demonstre sensitividade com relação aos pontos de vista de seus leitores e suas necessidade de dialogar com ele (Myers, 1989). Em outras palavras, a tentatividade pode expressar não apenas incerteza e reduzir a responsabilidade do escritor com relação às suas proposições, mas também pode facilitar a abertura de dialogo com o leitor.

Além dessas complicações, aprendizes passam por dificuldades porque significados epistêmicos podem ser sinalizados de diversas maneiras. Enquanto verbos modais como might, may, will e would têm recebido atenção proeminente na literatura como dispositivos utilizados para a expressar certeza e incerteza, pelo menos outros 350 itens lexicais também podem ser empregados para esse propósito (Holmes, 1988:27). Esses incluem: adjetivos (probable, certain, possible), verbos epistêmicos (believe, think, seem), substantivos (possibility, tendency) e advérbios (obviously, probably, indeed). Além disso, itens que expressam incerteza, tais como almost e about, podem modificar a força epistêmica de afirmações (Channel, 1994; Dubois, 1997) e expressões utilizadas para manipular definitividade, tais como usually e generally, também contribuem para as "escalas de probabilidade e usualidade às quais o termo modalidade estritamente pertence" (Halliday, 1985:86; minha tradução). Do mesmo modo, enquanto a expressão de comprometimento do escritor é um fenômeno notadamente lexical, orações condicionais, perguntas, marcadores de contraste e tempo verbal podem também ser utilizados para transmitir significados epistêmicos (Perkins, 1983).

As dificuldades dos aprendizes são agravadas pelo fato de que uma porção significativa desses dispositivos é ignorada ou mal interpretada em manuais de escrita acadêmica e livros de EFL (Hyland, 1994; Holmes, 1988). Freqüentemente os professores não ensinam aos aprendizes expressões que possam servir de alternativa para afirmações categóricas. A

\footnotetext{
${ }^{1}$ Definitividade é entendida aqui como quão definitiva uma proposição é. Nesse sentido, tem uma relação de sinonímia com o termo assertividade.
} 
falta de materiais pedagógicos adequados é de particular importância, porque escritores inexperientes não possuem um conhecimento suficiente de seus possíveis leitores. Bloor e Bloor (1991), por exemplo, consideram o grau de convicção permitido em escrita acadêmica um aspecto pertinente às diferenças culturais.

Resumindo, a necessidade de fazer proposições que sejam nem muito exageradas nem suaves demais em relação a fatos ou evidencias é uma área da escrita acadêmica que muitos aprendizes, tanto nativos quanto estrangeiros, acham desafiadora.

\section{DESCRIÇÃO DOS CORPORA E DO MÉTODO}

Este estudo utiliza dois pequenos corpora. O primeiro é uma coleção de composições escritas por alunos universitários brasileiros cursando a faculdade de Letras-Inglês no terceiro e no quarto ano de instrução. Br-ICLE ${ }^{2}$, como é chamado o componente brasileiro, possui hoje 33.794 palavras dispostas ao longo de 67 composições argumentativas. O segundo corpus, também com mais ou menos 34.000 palavras, é um subcorpus do Louvain Corpus of Native English Essays - LOCNESS, escrito por aprendizes britânicos e americanos de idade e nível de instrução similar aos dos aprendizes brasileiros ${ }^{3}$.

Pode-se presumir que existam muitas diferenças sociais e educacionais entre esses grupos, o que, por sua vez, talvez não permita uma comparação direta entre os seus textos. Isso deve ser levado em consideração quando considerarmos os resultados. Contudo, existem também muitas semelhanças, especialmente entre a idade e o nível de instrução dos aprendizes. Da mesma forma, os tópicos das composições não são idênticos, mas eles podem ser considerados comparáveis: ambos consistem de composições argumentativas cujos tópicos estão relacionados a questões sociais contemporâneas. A equivalência de gênero, tipo de texto e tópicos sugere a existência de pouca diferença com relação às necessidades impostas pela tarefa no que tange à expressão certeza ou incerteza. Assim, acredito que os resultados reflitam diferenças reais na maneira pela qual os dois grupos empregam esta característica pragmática em contextos autênticos de uso.

Para determinar a tipologia e freqüência de expressões lexicais de certeza e incerteza nestes dois corpora, uma lista de tais itens, comuns entre falantes nativos, foi preparada. A fonte principal foi a analise de Holmes (1988) da seção 'J' dos corpora de inglês escrito Brown e LOB (Lancaster/Oslo-Bergen). Isto foi suplementado por estudos de modalidade (Coates, 1983; Hyland 1996a; Perkins, 1983), gramáticas referenciais (Quirk et al, 1972)

\footnotetext{
2 Para informações adicionais consulte Sardinha (2000), Recski (2002), ou acesse o site www.lrecski.hpg.com.br

${ }^{3}$ LOCNESS é utilizado para comparar os diferentes componentes nacionais do International Corpus of Learner English - ICLE. O ICLE (Granger, 1993) é um projeto multinacional que busca identificar as características compartilhadas por uma grande variedade de interlinguas. Nesse projeto, cada subcorpus nacional contribui com cerca de 200 mil palavras (cerca de 400 composições argumentativas). Os alunos que contribuíram com suas composições para o ICLE compartilham os seguintes atributos. Eles são adultos (cerca de 20 anos de idade) que estão estudando inglês como língua estrangeira, i.e. eles são alunos de EFL, não de ESL. Seu nível de proficiência é 'avançado', noção que é definida da seguinte maneira: eles são estudantes de graduação em língua inglesa no terceiro ou quarto ano de instrução. Embora as composições produzidas envolvam uma variedade grande de tópicos o conteúdo é similar, haja visto que todos os tópicos são argumentativos e não técnicos.
} 
e estudos de expressões de freqüência (Kennedy, 1987) que podem ser utilizadas para ajustar a intensidade de afirmações (Halliday, 1994). Destas fontes, um inventário dos 75 itens lexicais epistêmicos mais freqüentes em escrita acadêmica de falantes nativos de inglês foi construído (vide Apêndice).

\section{RESULTADOS}

\subsection{Distribuição de freqüência de dispositivos epistêmicos}

O número total de itens lexicais empregados para expressar significados epistêmicos nos corpora aparece na Tabela 1. A análise revela semelhanças importantes na distribuição de frequiência, uma vez que ambos grupos utilizaram um dispositivo epistêmico a cada 56 palavras. Estes resultados estão de acordo com aqueles apresentados em artigos acadêmicos publicados (Skelton, 1988; Adams Smith, 1984).

Além de semelhanças na frequiência total, existem similaridades consideráveis de uso com will, would, believe, possible e always ocorrendo entre os seis itens mais freqüentemente empregados por ambos grupos, embora com diferenças significativas em termos de freqüência entre tais itens para os dois grupos (Tabela 2).

A Tabela 2 mostra, por exemplo, que would epistêmico é pelo menos duas vezes mais freqüente na amostra dos escritores nativos do que na dos brasileiros e que a freqüência de will é praticamente a mesma. Como esses dois modais podem ser usados para indicar possibilidades tanto no presente quanto no futuro, esta distribuição sugere diferenças conceituais, apontando-se que os escritores nativos preferem utilizar expressões mais provisórias ou tentativas através do uso de would. O uso de think como um verbo epistêmico é empregado quase exclusivamente para expressar o grau de certeza dos aprendizes em ambas populações, mas é quatro vezes mais frequiente no Br-ICLE. Informações disponíveis sobre escrita acadêmica de "experts" (Holmes, 1988) indicam que would, seem, will e may aparecem freqüentemente, o que, por sua vez, está de acordo com os itens encontrados em ambos grupos.

Tabela 1. Número total de itens lexicais empregados para expressar modalidade epistêmica.

\section{Br-ICLE TOCNESS Total}

$\begin{array}{cccc}\text { Total de dispositivos } & 618 & 603 & 1221 \\ \text { Ocorrências por } 100 \text { palavras } & 1.84 & 1.82 & 1.83\end{array}$

No geral, os itens lexicais empregados por ambos grupos são restritos em sua amplitude, sendo que os dez itens lexicais mais empregados por aprendizes brasileiros constituem $69 \%$ do total de itens empregados epistemicamente. Para os aprendizes nativos, esse dado corresponde a $82 \%$ do total. Apenas 39 dos 75 itens aparecem mais do que cinco 
RECSKI - Negociando certeza e incerteza...

vezes no LOCNESS e onze não ocorrem nenhuma vez. Tanto about quanto think ocorrem quatro vezes mais no corpus de aprendizes brasileiros do que no dos aprendizes nativos.

Tabela 2. Itens mais comumente empregados para expressar modalidade espistêmica

\begin{tabular}{|cccccc|}
\hline Ordem & $\begin{array}{c}\text { Br-ICLE } \\
\text { Item }\end{array}$ & Freqüiência & Ordem & $\begin{array}{c}\text { LOCNESS } \\
\text { Item }\end{array}$ & Freqüiência \\
\hline 1 & will & 108 & 1 & would & 172 \\
2 & think & 69 & 2 & will & 115 \\
3 & would & 68 & 3 & believe & 49 \\
4 & always & 31 & 4 & seem & 36 \\
5 & believe & 27 & 5 & may & 30 \\
6 & possible & 27 & 6 & pissible & 28 \\
7 & seem & 25 & 7 & always & 18 \\
8 & may & 24 & 8 & never & 16 \\
9 & sometimes & 24 & 9 & of course & 16 \\
10 & never & 22 & 10 & perhaps & 16 \\
Total & & 425 & Total & & 496 \\
\hline \hline
\end{tabular}

\subsection{Distribuição gramatical}

A Figura 1 mostra uma acentuada correlação no uso de verbos lexicais, adjetivos e substantivos empregados para expressar certeza e definitividade, mas também indica diferenças marcantes no uso de advérbios e verbos modais.

Ambos grupos empregam destacadamente os verbos modais - especialmente will, would e may - para expressar significados epistêmicos. Advérbios são mais freqüentemente empregados, inclusive com uma variedade maior, pelos aprendizes nativos, sendo encontradas 57\% a mais de ocorrências. Ambos grupos preferem advérbios a verbos lexicais como estratégias para a expressão de certeza e incerteza, embora esse último ocorra mais em textos acadêmicos publicados (Holmes, 1983; Hyland, 1996a).

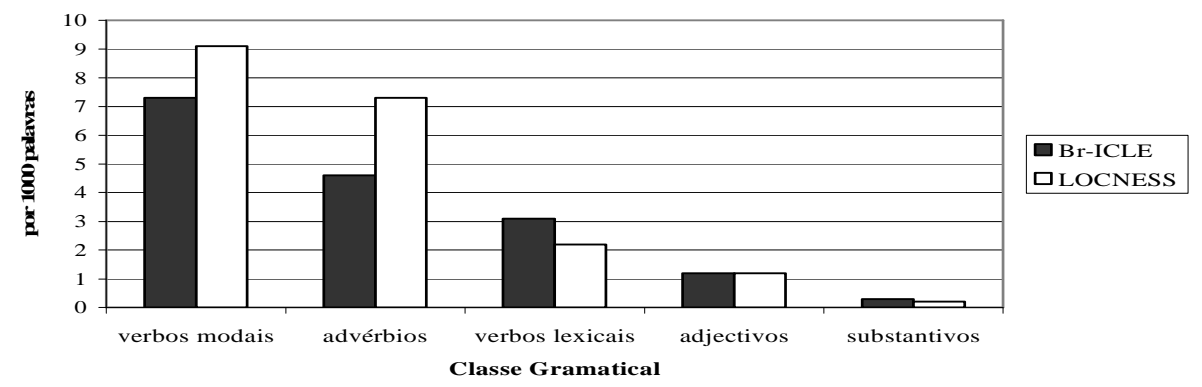

Figura 1. Frequiência Relativa de Classes Gramaticais empregadas para expressar modalidade epistêmica. 
A popularidade de advérbios semanticamente equivalentes às formas verbais pode estar relacionada à incerteza sobre como empregar verbos lexicais apropriadamente na construção de proposições. Verbos lexicais oferecem uma maneira mais precisa e 'aberta' de sinalizar a convicção do escritor do que advérbios e podem sinalizar graus relativos de certeza (1) e incerteza (2):

(1) I think this question is very relevant nowadays, because it is amazing how people (specially from the lower classes) keep having babies. (Br-ICLE)

(2) It seems to me that for the past few centuries, Britain has considered itself to be detached from the rest of Europe. (LOCNESS)

Além de modificar a força de uma afirmação, verbos também indicam se esta afirmação traz consigo uma forma de julgamento (3), ou oferecem uma justificativa através de evidências, indicando a confiabilidade da fonte da informação (4 e 5) (Hyland, 1996a).

(3) I believe life is a wonderful thing, that it is a God's blessing to be alive, and that life should be supported until the end (Br-ICLE)

(4) Demographists claim that it is a major factor in considering the problems of that country's evolution - for example ... (LOCNESS)

(5) This view does not seem to hold much credibility in Europe. (LOCNESS)

A habilidade de utilizar palavras adequadas para atribuir credibilidade a proposições relacionando-as a fatos, entretanto, parece ser uma tarefa difícil para ambas populações. Advérbios podem ser mais fáceis de serem manipulados com segurança por aprendizes. Eles não apenas são muito mais comuns na fala do que na escrita (Holmes, 1983), mas também permitem uma maior mobilidade dentro da oração e oferecem claras distinções escalares (Quirk et al, 1972:438). Isto permite que escritores intensifiquem ou diminuam seu comprometimento com a confiança de que eles estão utilizando uma estrutura acadêmica discursiva adequada sem correr o risco de erros oriundos de formas verbais inadequadas.

$\mathrm{O}$ fato de que aprendizes parecem preferir advérbios epistêmicos a verbos lexicais parece estar relacionado da tarefa de definir qual o tom adequado para a escrita acadêmica. De acordo com Smith (1986), o tom que deve ser empregado em gêneros mais marcadamente expositivos requer um distanciamento máximo entre o autor e o leitor e uma alta percentagem de construções passivas é normalmente citada como uma das características de uma boa escrita acadêmica em tais gêneros. Normalmente se ensina aos aprendizes que a escrita acadêmica requer impessoalidade, mas quando se escreve, isto é dificultado pela necessidade de avaliar a informação e acessar a sua validade. O uso de verbos epistêmicos adequados, portanto, envolve escolhas difíceis de léxico, tempo verbal e voz que possam trazer os efeitos retóricos desejados. Os advérbios oferecem aos aprendizes uma maneira muito mais simples de expressar atitude com relação às suas proposições e de ajustar a força de suas afirmações sem que seja preciso tais complicações gramaticais e lexicais.

Com base nas evidências das amostras, é possível sugerir que os aprendizes respondem a essas dificuldades não apenas mesclando um estilo formal e informal de escrever, mas também através da transferência de práticas conversacionais para os seus 
textos acadêmicos. Nenhum dos corpora é caracterizado por uma grande variedade de verbos lexicais: think, believe e seem correspondem a mais de dois terços de todos verbos epistêmicos em ambos corpora. O uso restrito de verbos epistêmicos e a preferência por expressões conversacionais aponta para a incerteza desses aprendizes sobre como empregar esses verbos de maneira eficiente em sua argumentação assim como sua dificuldade de compreender certas nuances deste registro acadêmico. Possuindo, aparentemente, um conhecimento limitado de práticas de escrita acadêmica formal, nenhum dos grupos é capaz de empregar esses verbos de forma irrepreensível ao fazer suas proposições. Para evitar violar certas expectativas acadêmicas, esses grupos procuram se basear mais naquilo que têm mais confiança: verbos modais e advérbios.

\subsection{Tipos de comprometimento epistêmico}

Dispositivos epistêmicos expressam diferentes graus de certeza com relação à validade da informação proposta; obviously, por exemplo, expressa um grau de certeza muito maior do que might. Para comparar o uso de modalidade nos dois corpora, categorias epistêmicas distintas foram estabelecidas para localizar certos padrões e itens lexicais em uma escala que se estende de certeza máxima a mínima. Enquanto, inevitavelmente, essa escala é arbitrária em alguns casos, tal categorização é compatível com aquelas sugeridas por outros pesquisadores na literatura da área. Categorias similares foram adotadas em gramáticas referenciais (Halliday, 1994; Quirk et al., 1972; Leech e Svartvick, 1994) e demonstraram ser úteis na análise empírica de vários corpora escritos e transcritos (Holmes, 1983, 1984).

A análise baseada nessas categorias revelou diferenças significativas com relação ao grau de certeza e tentatividade empregados pelos dois grupos de aprendizes (Figura 2). Os resultados parecem confirmar a expectativa de que a escrita acadêmica de muitos aprendizes brasileiros seja caracterizada por proposições mais assertivas, um tom mais autoritário e um comprometimento maior dos escritores do que o discurso dos aprendizes nativos. Em geral, os aprendizes brasileiros empregaram itens que expressam certeza $56 \%$ mais freqüentemente do que os aprendizes nativos enquanto esses empregaram $69 \%$ a mais itens que expressam probabilidade. Dispositivos que expressam possibilidade, freqüência e aproximação tiveram distribuições semelhantes.

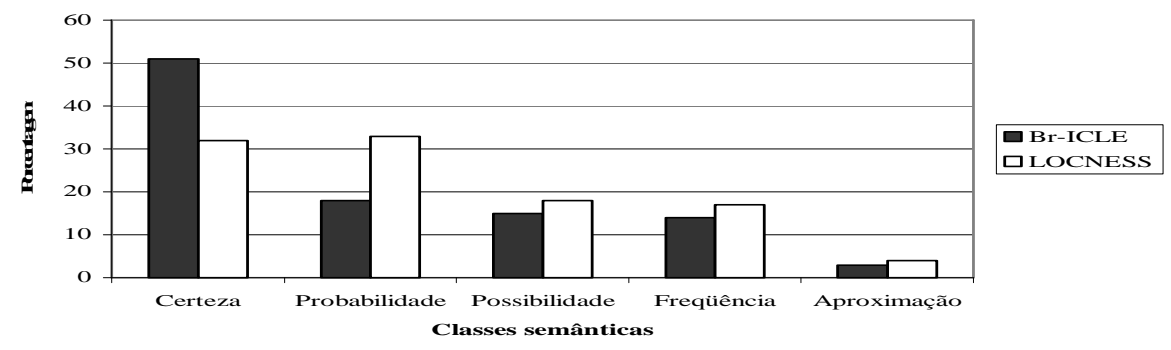

Figura 2. Distribuição de Categorias Epistêmicas (\%) 
O fato dos aprendizes brasileiros não moderarem suas proposições suficientemente pode estar relacionado a um conhecimento lingüístico inadequado, talvez causado por diferentes interpretações de formas semânticas equivalentes. Alternativamente, isto pode representar violações "sócio-pragmáticas" resultantes da interpretação errônea do que é o uso adequado da linguagem (Thomas, 1983). Isto é, enquanto tipicamente aprendizes podem ser mais indiretos ao escreverem em português, eles podem estar compensando em demasia aquilo que julgam ser maneiras de ser direto em inglês e não conseguem distinguir entre as convenções de diferentes tipos de textos. Assim, os aprendizes talvez selecionem uma estratégia que acreditam expressar uma locução socialmente apropriada, mas que na verdade expressa um grau inapropriado de assertividade e objetividade para um registro acadêmico.

Cerca de metade dos dispositivos epistêmicos empregados pelos aprendizes brasileiros servem para expressar certeza, como se pode observar nos exemplos que seguem:

(6) It can be concluded, obviously, that when politicians want something they do that. I mean when they are interested they approve whatever they want. (Br-ICLE)

(7) I am sure that the adults who have really enjoyed their childhood and their adolescence are much happier than who have been precocious and have not played as a child or agued as an adolescent. (Br-ICLE)

(8) Actually, poor people get sick much more often because they cannot have healthy eating habits and access to good doctors and medicine. (Br-ICLE)

(9) There are clear evidences that exists a certain standard of life, and it has always existed. (Br-ICLE)

Por outro lado, a maioria dos itens nas composições dos aprendizes nativos são utilizados para indicar que as proposições são provavelmente verdadeiras (10-12), ou para expressar incerteza com relação a validade das mesmas (13-15).

(10) The result is therefore riddled with contradictions which appear to remain unresolved by the government. (LOCNESS)

(11) The aims of such union are essentially economic. (LOCNESS)

(12) We are more likely to be involved in arguments which involve frustration with people we know and come in contact with. (LOCNESS)

(13) Amsterdam is seen as a cosmopolitan place and, therefore, the guilt that is portrayed there is perhaps a symbol of the guilt of mankind throughout the world. (LOCNESS)

(14) Since the ruling on euthanasia took place in 1988, it is possible that the worst in the debate on euthanasia is yet to come. (LOCNESS)

(15) Another reason is that inmates are more tense and aggressive around the time of an execution and that to televise it might cause violence. (LOCNESS)

Deve ser indicado, entretanto, que uma vez que procurei seguir outros pesquisadores ao estabelecer categorias epistêmicas discretas, os resultados devem ser tratados com alguma cautela. As categorias oferecem uma base importante para que possamos comparar a amplitude e freqüência dos itens empregados pelos diferentes grupos, mas existem sérias dificuldades em se mapear inequivocadamente o grau preciso de convicção de cada termo 
em cada oração. Expressões modais raramente são totalmente determináveis em termos de significado porque o valor semântico de qualquer termo está sempre sujeito a limitações pragmáticas que podem alterar o seu significado. $\mathrm{O}$ advérbio quite, por exemplo, pode tanto reduzir como intensificar a força de outra palavra dependendo do contexto onde ocorre. (Hyland, 1996d). A ocorrência de cada um dos 75 itens selecionados para este estudo foi, assim, cuidadosamente avaliada em seu contexto para assegurar que estava sendo utilizada para expressar um significado epistêmico. Isto significou, por exemplo, que exemplos homônimos de palavras tais como appear, possible e clear não foram contados e que muitas ocorrências de verbos modais foram excluídas onde estes expressavam significados deônticos. Além disso, verbos epistêmicos tais como know, think e believe foram assinalados como epistêmicos apenas quando antecedidos de um sujeito pessoal ou empregados para citar outro autor.

Mas contexto não é um fator desambiguador em todos os casos e lingüistas discordam com relação a certos aspectos cruciais. Coates (1983:177), por exemplo, argumenta que will epistêmico é empregado para predizer de maneira confiante eventos atemporais, no presente e no futuro. Entretanto futuridade envolve incerteza (Palmer, 1990) e Lyons (1977:809) sugere que introduzir qualquer modalidade em uma oração faz com que sua veracidade dependa do conhecimento, talvez limitado, do autor. Meu posicionamento é que, em uma escala epistêmica que vai de máxima a mínima certeza, will parece expressar uma avaliação de que uma proposição é válida na medida da certeza do autor. Portanto, sigo Leech e Svartvick (1994), Quirk et al (1972) e outros ao situar will na categoria certeza, reconhecendo que o seu efeito é normalmente 'intensificar' expressões de convicção do autor.

Desta forma, enquanto categorias epistêmicas buscam capturar os amplos significados semânticos de certos itens dentro de uma escala que vai de máxima a mínima certeza, admito que a categorização é incerta em alguns casos, tornando perigosas as quantificações precisas. Estas considerações devem ser levadas em conta ao analisarmos os dados a seguir.

\subsection{Expressões de Certeza e Incerteza Empregadas pelos Aprendizes}

Mais uma vez, a análise de categorias epistêmicas indica distribuições não uniformes entre os dois grupos. Os aprendizes brasileiros empregaram uma proporção significativamente maior de itens que indicam certeza enquanto probabilidade e possibilidade são mais freqüentes entre os aprendizes nativos. A qualificação de proposições por intermédio de dispositivos de aproximação tais como almost e to a certain extent é relativamente constante entre os dois grupos.

Essa tendência de aprendizes nativos modificarem suas proposições com expressões mais tentativas é claramente demonstrada na Figura 3. Como se pode observar, a Figura 3 mostra a categorização das formas em atos que fortalecem ou enfraquecem a força das proposições que foram feitas, i.e., o grau de expressão de certeza e incerteza do autor tanto para reduzir quanto para intensificar a força de uma proposição. Assim, as palavras never e always foram incluídas na categoria 'certeza' e todos as outras formas menos determinadas nas categorias 'probabilidade', 'possibilidade' e 'freqüência'. Pode-se notar que enquanto os aprendizes brasileiros empregam em geral menos dispositivos, sua escrita é caracterizada 
por proposições epistemicamente mais assertivas. A escrita dos aprendizes nativos, por outro lado, mostra um emprego maior de qualificações cautelosas com relação às suas proposições.

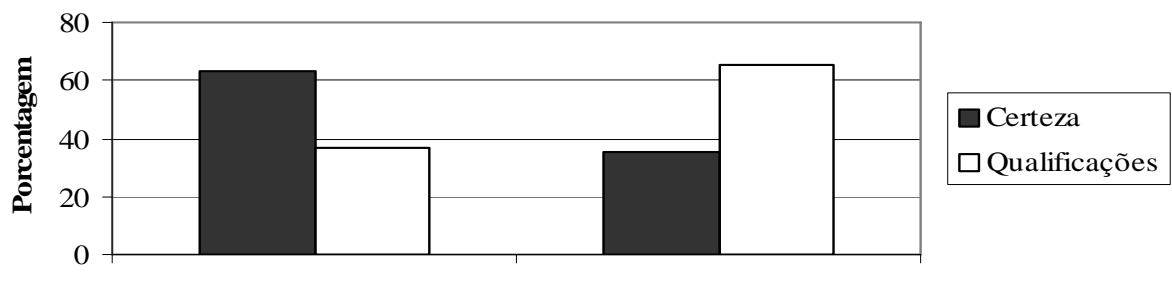

Br-ICLE

LOCNESS

Corpora

Figura 3. Distribuição de Itens Expressando Certeza e Qualificações entre os Corpora.

Apesar das diferenças em freqüência, existem semelhanças consideráveis entre os itens empregados pelas duas populações. A Tabela 3 mostra os itens mais freqüentes que ocorrem em cada categoria assim como a proporção que representam de cada categoria.

\begin{tabular}{|c|c|c|c|}
\hline Item & Br-ICLE & Total de ocorrências & LOCNESS \\
\hline \multicolumn{4}{|l|}{ A. Certeza } \\
\hline actually & 14 & & 9 \\
\hline certainly & 8 & & 18 \\
\hline indeed & 7 & & 14 \\
\hline in fact & 15 & & 7 \\
\hline know & 23 & & 2 \\
\hline think & 69 & & 11 \\
\hline will & 108 & & 115 \\
\hline \%dos iten de certeza & 91.4 & & 73.7 \\
\hline \multicolumn{4}{|l|}{ B. Probabilidade } \\
\hline beieve & 27 & & 49 \\
\hline probably & 13 & & 11 \\
\hline quite & 12 & & 8 \\
\hline seen & 25 & & 36 \\
\hline would & 68 & & 172 \\
\hline$\%$ dos itens de probabilidade & 72.5 & & 74.1 \\
\hline \multicolumn{4}{|l|}{ C. Probabilidade } \\
\hline may & 24 & & 30 \\
\hline might & 5 & & 13 \\
\hline perhaps & 2 & & 16 \\
\hline possible (ly) & 27 & & 28 \\
\hline$\%$ dos itens de possibilidade & 97.8 & & 96.3 \\
\hline \multicolumn{4}{|l|}{ D. Freqüuência } \\
\hline always & 31 & & 18 \\
\hline never & 12 & & 16 \\
\hline often & 10 & & 40 \\
\hline sometimes & 24 & & 4 \\
\hline usualy & 18 & & 12 \\
\hline \% dos itens de frequiência & 79.3 & & 68.5 \\
\hline
\end{tabular}

Tabela 3. Itens mais frequientes nas principais categorias epistêmicas 
Como pode ser visto, os mesmos itens constituem a grande maioria dos termos empregados em cada categoria, embora os aprendizes nativos pareçam empregar um vocabulário maior para expressar certeza e frequiência. Will é o indicador de certeza preferido por ambos grupos, ao passo que know, think e in fact são muito mais comuns entre os aprendizes brasileiros.

Existem inúmeras diferenças no grau de proficiência, especialmente no uso de termos que expressam certeza e freqüência. Várias formas tais como indeed, might, often e certainly são mais comuns nos textos dos aprendizes nativos. As composições dos aprendizes brasileiros, por outro lado, são dominadas por will, think e possible que constituem $36 \%$ de todos os itens epistêmicos empregados por este grupo. Na escala de freqüência, always e never constituem $50 \%$ de todas as formas empregadas por aprendizes brasileiros. Indicadores mais indeterminados de freqüência tais como usually e often são mais escassos nos textos dos aprendizes brasileiros e itens como generally e frequently são quase que exclusivamente encontrados apenas nos textos dos aprendizes nativos.

\subsection{Contrastando expressões personalizadas e impersonalizadas}

Um aspecto interessante de como escritores modificam suas proposições é o uso de expressões personalizadas e impersonalizadas (Leech e Svartvick, 1994). Expressões personalizadas envolvem explicitamente o autor e como esse acessa a validade de uma proposição através do uso de pronomes na primeira pessoa:

(16) I strongly believe that the limitation laws do not respect the Universal Declaration of Human Rights, written in 1948 by the United Nations General Assembly. (Br-ICLE)

(17) They get together and elaborate seven commandments that, to me, can be said to represent some sort of 'constitution'. (Br-ICLE)

Expressões impersonalizadas, por outro lado, ocultam o ponto de vista do autor quando ele comenta sobre a veracidade de uma proposição e tipicamente escondem a fonte de julgamentos epistêmicos através do uso de advérbios, pronomes impessoais ou voz passiva:

(18) It is evident that Voltaire utilizes both character and circumstance to tackle the problem of philosophical optimism and reveal his scorn of it to the public. (LOCNESS).

(19) Apparently, this will extend our free market economy to the whole of Europe, or at least to those countries which participate. (LOCNESS)

Como foi descrito acima, escolhas de voz e pessoa aliadas a expressões modais ajudam a estabelecer um tom acadêmico apropriado com um alto grau de formalidade entre o autor e o leitor. Como se pode suspeitar, o uso desses dispositivos difere de maneira significativa entre os aprendizes nos dois corpora. Os aprendizes brasileiros parecem transferir características de um registro mais pessoal para este gênero argumentativo e exibem uma menor consistência em suas escolhas, freqüentemente utilizando comentários epistêmicos de forma incongruente para o contexto acadêmico. Esses aprendizes empregam muito mais verbos epistêmicos antecedidos de pronomes na primeira pessoa do que os aprendizes 
nativos. Pronomes pessoais ocorrem com freqüência com verbos epistêmicos em escrita acadêmica publicada, onde eles caracteristicamente funcionam interpessoalmente para intensificar ou reduzir a força de uma proposição e o seu grau de generalização. Para muitos aprendizes, todavia, tentativas de intensificar uma convicção dessa maneira estão cheias de perigos:

(20) In my opinion, as far as I am concerned the biggest problem is the over population. (Br-ICLE)

(21) I am still trying to make something and I am very sure that we will save the planet. (Br-ICLE).

Portanto, enquanto a presença de expressões pessoais per se não indica um tom inapropriado, sua freqüência, incongruência e relativa informalidade nas composições dos aprendizes brasileiros sugere uma inconformidade com a formalidade esperada para o gênero que estão escrevendo.

Estes aprendizes também parecem ter mais dificuldades para manipular expressões impessoais apropriadamente em escrita acadêmica. Isto ocorre, particularmente, quando a intenção de suprimir sujeitos pessoais gera um conjunto de erros tais como o uso inadequado de sujeitos ocultos e escolhas temporais (22-23). Além disso, o uso de advérbios no inicio de orações para expressar certeza ou incerteza geralmente confunde registros falados com escritos (24-25):

(22) It is no doubt that the search for eternal youth is a common thing in our society. (BrICLE)

(23) It is clearly saying that in the next years we will find lots of graduate students in this situation, because everybody who has a job is doing everything to maintain it. (BrICLE)

(24) Obviously, this is the principal objective of media discourse to produce an ideological discourse replete of power and domination. (Br-ICLE)

(25) Probably, he gets very interested in specializing our doctors in this new technique of youthfulness. (Br-ICLE)

Outra maneira de se proteger é atribuir um ponto de vista a outra pessoa:

(26) The scientists argue that if the authorities do not anything, we will be running the risk of extinction. (Br-ICLE)

(27) On the other hand, some people claim that the criminals are a financial burden to society and keep them arrested is waste of public money. (Br-ICLE)

Tais expressões impessoais são comuns em artigos publicados (Thompson e Ye, 1991) e ajudam a protegem o autor na medida que repassam para outra fonte a responsabilidade sobre a validade de proposições. Neste estudo, os aprendizes nativos utilizaram formas impersonalizadas em quantidades e variedades bem maiores do que os aprendizes brasileiros. Parece ficar claro então, que enquanto esses usos demonstram uma tentativa de seguir as regras de objetividade e impessoalidade geralmente preconizadas 
por professores e livros didáticos, a necessidade de avaliar proposições em gêneros acadêmicos pode trazer problemas sérios de expressão para escritores não nativos.

\section{IMPLICAÇÕES PEDAGÓGICAS}

Ambas amostras apresentam um uso limitado de formas, mas a dificuldade em usar dispositivos epistêmicos apropriadamente é mais evidente na escrita dos aprendizes brasileiros. A maior competência apresentada pelos aprendizes nativos nesse aspecto sugere que eles tenham uma melhor compreensão de convenções discursivas, talvez por intermédio de um sistema de ensino que saliente mais tais dispositivos, ou por estarem mais atentos a padrões de linguagem em suas leituras.

É interessante que exercícios sejam planejados para ajudarem os aprendizes a desenvolver estratégias de distinção entre fatos observados e interpretações, bem como para usar um leque maior de itens que possam ser empregados de forma eficiente para expressar significados epistêmicos. As categorias sugeridas acima oferecem um ponto sensato de partida para chamar a atenção dos aprendizes sobre diferentes graus de probabilidade e podem constituir uma base para exercícios por intermédio dos quais aprendizes possam identificar itens que expressem certeza, probabilidade, possibilidade e freqüência.

O domínio de várias formas e padrões para cada categoria epistêmica é essencial para uma maior variedade de expressões. Preferencialmente, os itens lexicais selecionados para o ensino deveriam, portanto, incluir aqueles que possam ser empregados em vários padrões (voz passiva, voz ativa, sujeitos construídos com "it”, etc.), que ocorram em diferentes classes gramaticais (certainly, likely, possibility) e que formem colocações (seems adequate, might suggest, appears unlikely). Este estudo sugere alguns dos itens mais freqüentes e mais produtivos em cada categoria. Assim, poder-se-ia discutir com nossos aprendizes os efeitos epistêmicos que obtemos ao remover itens de um determinado texto substituindo-os por itens de outras categorias. Reflexões acerca da relevância de expressões epistêmicas podem também ser motivadas permitindo que os aprendizes julguem os efeitos de reformulações que alterem o grau de certeza de um texto.

Os aprendizes também precisam de oportunidades para praticar o uso de diferentes formas. Reescrever exercícios que envolvam a substituição de expressões de certeza por expressões mais provisórias, por exemplo, comprovadamente constituem uma maneira eficiente de ajudar aprendizes a dominar a difícil noção de 'tentatividade' em escrita acadêmica (Salager-Meyer, 1994). Outras tarefas produtivas incluem a paráfrase de textos que envolvam diferentes graus de certeza e a utilização de atividades de escrita que explorem futuridade pessoal, política, social, etc (Hyland, 1996b). Certamente existe uma variedade de recursos pedagógicos para auxiliar os aprendizes a desenvolver um repertório de dispositivos epistêmicos que possam ser empregados com confiança em sua escrita acadêmica. Entretanto, é importante que perspectivas de ensino salientem não apenas a importância destes itens epistêmicos. Estes métodos também devem fornecer estratégias 
alternativas a padrões sintáticos complexos e ao uso demasiado de verbos modais que comumente predominam em livros didáticos e tornam modalidade tão difícil de ser aprendida.

\section{CONSIDERAÇÕES FINAIS}

De forma geral, os dados indicam que enquanto os dois grupos de aprendizes utilizam preponderantemente uma pequena variedade de itens (principalmente verbos modais e advérbios), a manipulação de certeza e afetividade em escrita acadêmica é particularmente problemática para os aprendizes brasileiros. Esses aprendizes empregam construções sintáticas mais simplificadas, utilizam uma variedade menor de dispositivos epistêmicos, são muito mais assertivos em suas proposições e têm maiores problemas para expressar um grau adequado de certeza. Acredito que esta falta de familiaridade com as convenções de vários gêneros acadêmicos em inglês possa ser prejudicial para as oportunidades acadêmicas e profissionais destes aprendizes. Isto porque esses erros freqüentemente influenciam a maneira como os leitores julgam a coerência e compreensão desses textos, o impacto de seus argumentos e a competência acadêmica de seus autores.

É claro que este estudo abrange apenas um grupo de aprendizes brasileiros, mas a literatura sugere que é improvável que esses aprendizes difiram acentuadamente de outros aprendizes em suas dificuldades de expressar certeza e incerteza em inglês. Muitas práticas de ensino da língua inglesa, contudo, têm negligenciado a importância da linguagem epistêmica e os tipos de dificuldades reveladas neste estudo podem ser - ao menos parcialmente - atribuídas a essa negligência. Com um número crescente de alunos de diversos países freqüentando cursos universitários em países de língua inglesa, é essencial que essas características do discurso acadêmico sejam disponibilizadas para estes alunos. Talvez assim eles possam assumir um controle mais preciso dessa área de competência retórica fundamental para uma melhor comunicação em tais ambientes.

\section{REFERÊNCIAS BIBLIOGRÁFICAS}

ADAMS SMITH, D. (1984). Medical Discourse: Aspects of Author's Comment. The ESP Journal, 3: 25-36.

BIBER, D. ; CONRAD, S. ; REPPEN, R. (1998). Corpus Linguistics: Investigating language structure and use. Cambridge: CUP.

BLOOR, M.; BLOOR, T. (1991). Cultural expectations and socio-pragmatic failure in academic writing. In P Adams, B. Heaton \& P. Howarth (eds.), Socio-cultural issues in English for academic purposes (pp. 112). Basingstoke: Modern English Publications/British Council.

CHANNEL, J. (1994). Vague language. Oxford University Press.

COATES, J. (1983). The Semantics of the Modal Auxiliaries. Beckenham: Croom Helm. (1987). Epistemic modality and spoken discourse. Transactions of the Philological Society, 85: 100131. 
RECSKI - Negociando certeza e incerteza...

CRISMORE, A. (1990). Metadiscourse and Discourse Processes: Interactions and Issues. Discourse Processes, 13: 191-205.

DUBOIS, B. (1987). Something in the order of around forty to forty four: Imprecise numerical expressions in biomedical slide talk. Language in Society, 16: 527-541.

GRANGER, S. (1993). New insights into the learner lexicon: A preliminary report from the international corpus of learner English. In. L. Flowerdew \& K.K. Tong (eds.), Entering text. (pp. 102-113). Hong kong: Hong Kong University of Science and Technology.

HALLIDAY, M. (1985). An Introduction to Functional Grammar. London: Pinter. (1994). An Introduction to Functional Grammar (2nd ed.). London: Pinter.

HAMP-LYONS, L. (1991). The writer's knowledge and our knowledge of the writer. In. L. Hamp-Lyons (ed.) Assessing second language writing in academic contexts (pp. 51-68). Norwood, NJ: Ablex.

HOLMES, J. (1983). Speaking English with the appropriate degree of conviction. In C. Brumfit (ed.), Learning and Teaching Languages for Communication: Applied Linguistics Perspectives (pp. 100-121). London: CILT.

. (1984). Modifying illocutionary force. Journal of Pragmatics, 8: 345-365.

(1988). Doubt and certainty in ESL textbooks. Applied Linguistics, 9: 21-44.

httpllwww.hpg.Irecski.com.br

HYLAND, K. (1994). Hedging in academic writing and EAP textbooks. English for Specific Purposes, 13: 239-256.

. (1996a). Talking to the academy: Forms of hedging in science research articles. Written Communication, 13(2): 251-281.

(1996b). Nurturing hedges in the ESP curriculum. System, 24: 477-490.

(1996c). Writing without conviction? Hedging in Science Research Articles. Applied Linguistics, 17: 433-453.

(1996d). "I don't quite follow": Making sense of a modifier. Language Awareness, 5(2): 91-109.

KENNEDY, G. (1987). Quantification and the use of English: a case study of one aspect of the language learners' task. Applied Linguistics, 8(3): 264-286.

LEECH, G; SVARTVICK, J. (1994). A communicative grammar of English, $2^{\text {nd }}$ ed. London: Longman.

LYONS, J. (1977). Semantics, Vol. 1 \& 2. Cambridge: CUP.

MYERS, G. (1989). The pragmatics of politeness in scientific articles. Applied Linguistics, 10(1): 1-35.

PALMER, F. (1990). Modality and the English modals, $2^{\text {nd }}$ ed. London and New York: Longman.

PERKINS, M. (1983). Modal Expression in English. London: Pinter.

QUIRK, R; GREENBAUM, S; LEECH, G; SVARTVICK, J. (1972). A grammar of contemporary English. Harlow, Essex: Longman.

RECSKI, L. (2002). Computer-assisted error analysis: A study of prepositional errors in the Brazilian subcomponent of the International Corpus of Learner English (Br-ICLE). Masters Thesis. Florianópolis, Brazil: PGI/Universidade Federal de Santa Catarina. 
Trabalhos em Lingüística Aplicada (43) - Jan./Jun. 2004

SALAGER-MEYER, F. (1994). Hedges and textual communicative function in medical English written discourse. English for Specific Purposes, 13: 149-170.

(1998). Language is not a physical object. English for Specific Purposes, 17: 295-301.

(2000). Procustes' recipe: hedging and positivism. English for Specific Purposes, 19: 175-187.

SARDINHA, T. (2000). O corpus de aprendiz BR-ICLE. DIRECT Papers 40. São Paulo: LAEL - PUC/SP.

SKELTON, J. (1988). The care and maintenance of hedges. ELT Journal, 41: 37-43.

SMITH, E. (1986). Achieving impact through the interpersonal component. In B. Couture (ed.), Functional approaches to writing: Research perspectives (pp. 108-119). Norwood: Ablex.

STUBBS, M. (1986). A matter of prolonged fieldwork: Notes towards a modal grammar of English. Applied Linguistics, 7: 1-25.

THOMAS, J. (1983). Cross-Cultural Pragmatic Failure.

plied

Linguistics,

12(4): 365-382.

VANDE-KOPPLE, W. (1988). Metadiscourse and the recall of modality markers. Visual Language 22(2/3): 233-272. 


\section{APÊNDICE}

Compilado a partir das análises de Holmes $(1983,1988)$ e Kennedy (1987) das seções J dos corpora Brown e LOB; do corpus de artigos de biologia de Hyland (1996a); de gramáticas e de literatura em modalidade incluindo Coates (1983, 1987); Leech \& Svartvick (1994); Perkins (1983); Skelton (1988); Quirk et al (1972); Lyons (1977).

Adjetivos
apparent
certain
a certain extent
clear
evident
possible
probable
Advérbios
about
actually
almost
always
(not) always
apparently
approximately
around
certainly
clearly
definitely
doubtless
essentially
evidently
frequently
generally
in fact
indeed

largely
likely
never
normally
obviously
of course
often
perhaps
possibly
presumably
probably
quite
rarely
relatively
sometimes
surely
undoubtedly
usually
Substantivos
claim
doubt
estimate
evidence
possibility
Verbos lexicais
appear
argue

assume
believe
claim
doubt
estimate
expect
indicate
know
predict
presume
propose
seem
speculate
suggest
suppose
tend
think
Verbos modais
could
couldn't
may
might
should
shouldn't
would
wouldn't
will
won't

